no differences in age, weight, height or unadjusted BMD at the femoral neck between the depressed and nondepressed men $(P=0.08,0.34,0.41$ and 0.13 , respectively), but unadjusted BMD at the PA spine was significantly lower in those with an LHX $\left(1.254 \pm 0.187\right.$ vs. $\left.1.293 \pm 0.194 \mathrm{~g} / \mathrm{cm}^{2}, P=0.017\right)$. Age, weight and smoking-adjusted BMD were $2.8 \%$ lower at the PA spine $(1.255 \pm 0.015$ vs. $1.292 \pm$ $\left.0.006 \mathrm{~g} / \mathrm{cm}^{2}, P=0.025\right)$ and $3.0 \%$ lower at the femoral neck $\left(0.971 \pm 0.011\right.$ vs. $1.001 \pm 0.004 \mathrm{~g} / \mathrm{cm}^{2}$, $P=0.007)$ in those with an LHX compared with those nondepressed. Adjusting for selective serotonin reuptake inhibitors use did not affect these relationships.

Conclusion: These data are consistent with previous findings of diminished BMD in people with depressive disorders and symptoms and suggest that depression may be a risk factor for reduced BMD in communitydwelling adult men.

\section{Facial emotion processing in psychosis}

\section{N Joshua, A O'Regan, S Rossell}

Mental Health Research Institute of Victoria, Melbourne, Australia

Background: Investigations into facial affect processing in schizophrenia have produced variable results; some studies indicate no differences in performance, while others have shown impaired performance in patients. Variations in methodology such as the use of different stimuli, different target emotions and differing levels of task difficulty may be responsible for these inconsistent findings. The current study investigated task performance on four emotion-processing tasks in two different groups of patients with psychosis and a group of healthy controls. All four tasks used the same stimuli and target emotions but manipulated task difficulty.

Method: Age- and premorbid-IQ-matched patients with schizophrenia $(n=37)$, patients with bipolar disorder $(n=32)$ and healthy controls $(n=45)$ completed the affect discrimination, name affect, select affect and match affect subtests from the Comprehensive Affective Testing System (CATS; Froming et al. 2003).

Results: Results indicated no significant differences in performance on the affect discrimination and name affect subtests. On the select affect subtest, patients with schizophrenia were significantly impaired compared with both other groups. On the match affect subtest, patients with schizophrenia were the most impaired, followed by the bipolar group.

Conclusions: The results indicated that as task complexity increases, performance decreases, making the difference between psychosis groups and healthy controls more evident, with patients with schizophrenia showing the greatest impairment. Some subsets of the CATS may be insensitive to the subtle differences in performance between groups. Future studies should explore facial emotion processing with greater number of stimuli and include a facial processing control task.

\section{The SHADE project: self-help for alcohol/other drug use and depression \\ F Kay-Lambkin', V Carr', T Lewin', B Kelly², A Baker'}

${ }^{1}$ Centre for Mental Health Studies, University of Newcastle; and ${ }^{2}$ Centre for Rural and Remote Mental Health, Orange, Australia

Background: The co-occurrence of depression and alcohol/other drug misuse is more common than expected by chance alone. Despite this, an effective program of treatment is yet to be established for people experiencing this comorbidity. This is a concern, given rates of depression and alcohol misuse are on the increase.

Aim: This paper will report on the posttreatment alcohol/other drug- and depression-related outcomes of the SHADE project, a large-scale, multisite study of computerized psychological treatment.

Methods: SHADE participants were those with current levels of depression and current problematic use of alcohol, cannabis or amphetamines. Following an initial assessment, participants received one faceto-face case formulation session with a therapist and were subsequently randomized to receive nine sessions of SHADE therapy by a therapist, nine sessions of SHADE therapy through a computer or nine sessions of person-centered (supportive) counseling. Follow-up occurred at posttreatment, 6- and 12-month follow-up.

Results: Posttreatment results will be reported for the 250 participants recruited to the study in rural/remote and urban New South Wales.

Conclusions: Computerized treatment is not meant as a stand-alone therapy. The results from this study suggest that computer-based interventions can produce important gains for people with depression and alcohol/other drug use comorbidity. Further implications will be discussed.

\section{Duloxetine in the treatment of melancholic depression}

\section{K Kelin}

Eli Lilly Australia Pty Ltd, Australia 
Background: Treatment response for melancholic patients may differ from nonmelancholic depressed patients, with broader spectrum antidepressant drugs being required for the melancholic subtype. Outpatients $(n=202)$ meeting the diagnostic criteria for major depressive disorder (DSM-IV), with HAMD17 $\geq 20$ and melancholic features, participated in an 8-week, multicenter, open-label, naturalistic, variable dose pilot study (F1J-AY-HMCZ).

Method: All patients received duloxetine $60 \mathrm{mg} /$ day for the first 4 weeks. In patients not responding by this time, effectiveness of duloxetine treatment was compared between patients who either continued on $60 \mathrm{mg}$ or received a higher, flexible $(90-120 \mathrm{mg} /$ day) dose, for a second 4-week period.

Results: After the initial 4-week period, $40.6 \%$ of patients $(n=82)$ responded $(\geq 50 \%$ reduction from baseline HAMD17) to duloxetine $60 \mathrm{mg} /$ day, and $17.8 \%(n=36)$ achieved formal remission (HAMD17 $\leq 7)$ of symptoms. For nonresponders at 4 weeks, no significant differences were detected across the $60-\mathrm{mg}$ and 90 - to $120-\mathrm{mg}$ treatment groups in the rates of response $(47.9 \%$ and $42.9 \%$, respectively) or remission $(22.9 \%$ and $18.4 \%)$ over the next 4 weeks. Overall, $58 \%$ of enrolled patients responded to treatment by study completion. Nausea, headache, dry mouth and constipation were the most frequently reported emergent adverse events.

Conclusions: The overall response rates after 4 and 8 weeks were encouraging for this sample of melancholic patients. In patients who did not respond to duloxetine $60 \mathrm{mg}$ over the first 4 weeks of treatment, increasing to a higher dose in the second month was not associated with any greater clinical benefit.

\section{Censoring of prescribable doses: the case of risperidone long-acting injection}

\section{T Lambert}

OPEN/ORYGEN, University of Melbourne, Melbourne, Australia

Background: Suggested dose ranges for new medicines often prove to be different from those that are empirically derived in the field. The release of the first long-acting SGA (risperidone CONSTA) suggests that recommended doses are, in the main, too low and the highest dose does not allow the full spectrum of doses that are seen with the oral equivalent. To investigate this, recommended CONSTA doses (in risperidone oral equivalents) are compared with the distribution of doses found in a large pharmacoepidemiological database.
Methods: From the OPEN/SEER pharmacoepidemiological data archives. Mean doses of SGAs are examined over time and most likely mean stable doses calculated. These are compared with projected mean doses and are further compared with suggestions based on the Seeman's radioligand-free Kis. CONSTA is examined in particular.

Results: Projected final mean doses are summarized with respect to estimated near-maximal effective doses (Davis et al. 2003). With respect to CONSTA, the range of doses appears to be equivalent to only half of that available with the oral form.

Conclusions: This suggests that careful attention needs to be paid to patient selection. The current selection guidelines take in to account the censored dose range and it is suggested that this may influence those correctly assigned to therapy with this new medication form. It also suggests that the empirical finding that a proportion of patients require doses higher than the recommended maximum is consistent with known RISe distributions in clinical practice.

\section{Evolving trends in antipsychotic use in two major centers: 1998-2006}

\section{T Lambert ${ }^{1,2}$, T Callaly ${ }^{3}$, B Sing ${ }^{2}$}

'OPEN, OPEN/ORYGEN; ${ }^{2 T h e}$ University of Melbourne, Melbourne, Australia; and ${ }^{3}$ Barwon Health, Geelong, Australia

Background: In the past decade, there has been an evolution of psychiatric services, moving to a community model with the closing of mental hospital beds by 2000. During this period, new, arguably more efficient antipsychotics have been introduced. There are no longitudinal studies available of antipsychotic use based on individual subject case audits. This paper presents a pharmacoepidemiological perspective on this issue, comparing two large mental health service entities subject to the same statewide 'Framework' document for service provision and yet with unique prescribing philosophies.

Methods: Using a purpose-built database, prescribing information, sociodemographics and legal status data were collected from Australia, New Zealand and Asia. Data were acquired from community-treated patients or out patients. Data for two major centers in Victoria, Geelong and Northwestern MH network, were abstracted and analyzed with respect to their comparative usage trends. This analysis is performed for those with schizophrenia and those with bipolar disorder.

Results: The data indicate that although both centers evolved their prescribing as deinstitutionalization progressed, their disparate prescribing patterns 